\title{
Detección de cepas de Neisseria meningitidis resistentes a rifampicina en el Uruguay
}

\author{
Gabriel Pérez Giffoni, ${ }^{1}$ Gabriela García Gabarrot, ${ }^{1}$ \\ Adriana Alfonso, ${ }^{2}$ Mónica Pujadas ${ }^{3}$ y Teresa Camou ${ }^{1}$
}

Forma de citar Pérez Giffoni G, García Gabarrot G, Alfonso A, Pujadas M, Camou T. Detección de cepas de Neisseria meningitidis resistentes a rifampicina en el Uruguay. Rev Panam Salud Publica. 2011;30(6):540-4.

RESUMEN El objetivo de este trabajo fue caracterizar fenotípica y genotípicamente dos aislamientos de Neisseria meningitidis resistentes a rifampicina relacionados con dos eventos independientes de transmisión de enfermedad meningocócica grave que se presentaron en septiembre y octubre de 2010 en Montevideo, Uruguay. Se revisó también la base de datos de la vigilancia nacional de resistencia a los antimicrobianos de los últimos 10 años, para estimar la frecuencia de la particularidad de los meningococos caracterizados. La resistencia a rifampicina se estudió por el método epsilométrico. El serotipo y serosubtipo de los aislamientos se determinaron por ELISA y la caracterización genotípica se realizó por digestión del ADN con NheI y electroforesis en gel con campo pulsátil. Ambos aislamientos eran idénticos, B:2a:P1.5, y su fenotipo no figuraba en la colección de 408 cepas de N. meningitidis aisladas en el Uruguay en los últimos 10 años, con la excepción de dos aislamientos sensibles a rifampicina. Los dos aislamientos estudiados también compartían un pulsotipo único, diferente del de otros dos aislamientos resistentes a rifampicina obtenidos en 2003 y 2007. Por lo tanto, ambos eventos de transmisión fueron causados por una única cepa resistente a rifampicina, que podría haberse introducido al país desde otras regiones o haberse originado por un cambio del serogrupo $C$ al B, como producto de la presión selectiva ejercida por vacunas administradas a la población. Es necesario mantener y extremar la vigilancia. No obstante, en vista de que hasta el momento este tipo de hallazgo ha sido esporádico, no se justifica cambiar el fármaco antimicrobiano que se administra a los contactos para la profilaxis, a menos que se identifique un caso secundario.

Palabras clave Neisseria meningitidis; rifampin; meningitis; resistencia a medicamentos; farmacorresistencia microbiana; Uruguay.

La recomendación de iniciar de forma inmediata la administración de quimioprofilaxis a los contactos cercanos de cada caso de enfermedad meningocócica para prevenir los casos secundarios es

\footnotetext{
Ministerio de Salud Pública, Departamento de Laboratorios de Salud Pública, Montevideo, Uruguay. La correspondencia se debe dirigir a Teresa Camou, tcamou@chasque.net

2 Ministerio de Salud Pública, Departamento de Vigilancia en Salud, Montevideo, Uruguay.

3 Ministerio de Salud Pública, División de Epidemiología, Montevideo, Uruguay.
}

universalmente aceptada y se aplica en la mayor parte de los países. La definición de contactos cercanos se refiere a las personas que comparten el hogar con el caso y otras con un grado equivalente de contacto. Cuando se trata de niños en jardines infantiles, no hay unanimidad acerca de la quimioprofilaxis, ya que en algunos países se recomienda iniciarla después del primer caso y en otros, solamente después del segundo caso (1). Desde la década de 1960, el régimen más comúnmente administrado ha sido rifampicina cada 12 horas durante dos días, aunque también se han utilizado con éxito ciprofloxacina y ceftriaxona.

Ya en 1971 se dio a conocer la colonización faríngea por cepas de Neisseria meningitidis resistentes a rifampicina en contactos sometidos a quimioprofilaxis con ese antibiótico; ese hecho ha continuado documentándose desde entonces. También es posible observar un rápido desarrollo de resistencia in vitro 
al exponer cepas de meningococos a la rifampicina. El alto grado de resistencia es causado por mutaciones puntuales del gen $r p o B$, que codifica la subunidad $ß$ de la ARN polimerasa (2). Sin embargo, la resistencia a rifampicina entre casos clínicos secundarios de enfermedad meningocócica se ha notificado muy esporádicamente $\mathrm{y}$, en general, el aislamiento del caso índice ha correspondido a una cepa sensible (3). En los Estados Unidos de América tal situación solo se ha notificado en tres oportunidades (4).

El Uruguay participa en el Sistema de Redes de Vigilancia de los Agentes Bacterianos Responsables de Neumonías y Meningitis, SIREVA II, coordinado por la Organización Panamericana de la Salud (OPS), para llevar a cabo la vigilancia de cepas de Streptococcus pneumoniae, Haemophilus influenzae y $N$. meningitidis en el laboratorio (5).

El análisis de 3598 aislamientos de N. meningitidis obtenidos por los países participantes de SIREVA II entre $2000 \mathrm{y}$ 2005 detectó solamente $23(0,6 \%)$ cepas con resistencia intermedia a rifampicina y $0,2 \%(n=6)$ de cepas resistentes (5).

En el Uruguay, la enfermedad meningocócica es de denuncia obligatoria desde 1961. El Departamento de Vigilancia en Salud del Ministerio de Salud Pública realiza la investigación epidemiológica de los casos y aplica las medidas de quimioprofilaxis a los contactos, que consisten en la administración de rifampicina durante 48 horas. A su vez, el Departamento de Laboratorios de Salud Pública realiza la confirmación y tipificación de la totalidad de los aislamientos causantes de enfermedad invasiva provenientes de laboratorios de microbiología clínica de todo el país.

El objetivo de este estudio fue caracterizar fenotípica y genotípicamente dos aislamientos de $N$. meningitidis resistentes a rifampicina relacionados con dos eventos de transmisión de enfermedad meningocócica grave ocurridos en septiembre y octubre de 2010 en Montevideo, Uruguay. También se hizo una revisión retrospectiva de la base de datos sobre aislamientos obtenidos de enfermedad meningocócica invasiva durante los últimos 10 años, con el fin de estimar la frecuencia de la particularidad fenotípica de los meningococos relacionados con los dos eventos de transmisión de 2010. Los aislamientos habían sido identificados por pruebas convencionales y por galerías comerciales (Neisseria $4 \mathrm{H}$,
BioRad; Crystal, Becton Dickinson; API $\mathrm{NH}$, BioMérieux). La concentración inhibitoria mínima (CIM) de rifampicina se determinó por el método epsilométrico (E-test, BioMérieux) y se interpretó de acuerdo con las recomendaciones del Instituto de Estándares Clínicos y de Laboratorio (CLSI, por su sigla en inglés) (6).

La determinación de serotipo y serosubtipo se realizó por ELISA con el método modificado de Abdillahi y Poolman (7). Los extractos crudos de células enteras fueron fijados sobre microplacas e incubados con 19 sueros monoclonales (Instituto Nacional de Salud Pública y Protección Ambiental de Holanda o RIVM). Los anticuerpos que permanecieron unidos después de los lavados fueron revelados con conjugado antirratón de streptavidina peroxidasa biotilinado (DAKO) y O-fenilendiamina dihidroclorídica como sustrato. Las concentraciones óptimas de los sueros monoclonales habían sido determinadas con cepas de referencia y oscilaban entre $1 / 40$ y $1 / 500$.

El ADN de los aislamientos fue digerido por la enzima de corte poco frecuente NheI. Los megafragmentos obtenidos se separaron por electroforesis en gel con campo pulsátil (PFGE, por su sigla en inglés) por el método modificado de Popovic et al. (8). Los perfiles de bandas denominados pulsotipos, fueron clasificados en a) idénticos, b) genéticamente relacionados, si tenían de una a tres bandas de diferencia y c) no relacionados, si tenían más de tres bandas de diferencia.

Se utilizaron dos cepas de referencia como control: la cepa del serogrupo B, $\mathrm{H} 44$ /76, que ha sido totalmente secuenciada (9) y la cepa 1103 del serogrupo C, que había sido caracterizada como representativa de un clon que se diseminó en el Uruguay en la década de 1990 (10).

El caso índice del primer evento de transmisión, en septiembre de 2010, se trató de un paciente de 14 años de edad, con diagnóstico clínico de meningitis supurada. El cultivo del líquido cefalorraquídeo (LCR) fue negativo, aunque presentaba valores citoquímicos compatibles con meningitis bacteriana. Por lo tanto, se resolvió administrar inmediatamente quimioprofilaxis con rifampicina a los contactos. A las 48 horas de finalizado el tratamiento, el hermano menor del caso índice, de 3 años de edad, fue hospitalizado con púrpura fulminante y falleció. Del cultivo del LCR y de la sangre se obtuvo un meningococo se- rogrupo $\mathrm{B}$, resistente a rifampicina, con $\mathrm{CIM} \geq 32 \mu \mathrm{g} / \mathrm{ml}$.

El segundo evento de transmisión se presentó en octubre de 2010. Se trató de un paciente de 3 años de edad, con diagnóstico clínico de meningitis supurada. Del LCR se aislaron cepas de $N$. meningitidis serogrupo $\mathrm{B}$, resistentes a rifampicina, con CIM $\geq 32 \mu \mathrm{g} / \mathrm{ml}$. En el momento de su ingreso al centro hospitalario, su madre, de 23 años de edad, presentó convulsiones y fue traslada a la unidad de cuidados intensivos con diagnóstico de meningitis supurada; permaneció internada más de un mes. En este segundo caso, el cultivo del LCR obtenido después del inicio del tratamiento con antimicrobianos fue negativo.

La investigación epidemiológica no permitió identificar factores en común entre los dos eventos de transmisión, excepto por el hecho de que los pacientes provenían de Montevideo (aunque no de barrios cercanos) y que su condición socioeconómica era baja.

Los dos aislamientos resistentes a rifampicina eran B:2a:P1.5 (cepas 1847 y 1852). Con el fin de determinar la frecuencia de ese serotipo/serosubtipo entre los aislamientos de los serogrupos B y C, se analizó la colección de los últimos 10 años (cuadro 1). Así, se identificó el serotipo/serosubtipo 2a:P1.5 en 6 de los 14 aislamientos del serogrupo C, mientras que entre los 368 aislamientos del serogrupo B, se identificaron solamente 2 (2008 y 2010), además de los 2 ya estudiados. En suma, el serotipo/ serosubtipo 2a:P1.5 fue identificado en meningococos C y B con una frecuencia de menos de un aislamiento por año; representa 2,5\% de los 408 aislamientos analizados.

En cuanto a la susceptibilidad a rifampicina, se encontró un único aislamiento resistente en 2003, con una CIM $\geq 32 \mu \mathrm{g} / \mathrm{ml}$, correspondiente al serotipo/ serosubtipo B:15:P1.16 (cepa 1325) y otro con resistencia intermedia en 2007, con CIM=1 (cepa 1648), serotipo/serosubtipo B:7,1:NST, por lo tanto, diferente de los aislamientos de 2010.

Los aislamientos resistentes a rifampicina de 2010 se estudiaron por PFGE junto con los aislamientos de 2003 y 2007. También se analizaron las cepas control H44/76 del serogrupo B y 1103 del serogrupo $\mathrm{C}$ y otros cuatro aislamientos B y C de 2010. Los megafragmentos digeridos con la enzima NheI generaron siete perfiles de bandas diferentes en 
CUADRO 1. Número de aislamientos de Neisseria meningitidis por serogrupo y por año, Uruguay, 2001 a 2010

\begin{tabular}{|c|c|c|c|c|c|c|c|c|c|c|c|}
\hline \multirow[b]{2}{*}{ Año } & \multicolumn{2}{|c|}{$\mathrm{C}: 2 \mathrm{a}: \mathrm{P} 1.5$} & \multicolumn{2}{|c|}{ Serogrupo C Otros } & \multicolumn{2}{|c|}{ B:2a:P1.5 } & \multicolumn{2}{|c|}{ Serogrupo B Otros } & \multicolumn{2}{|c|}{ Otros serogrupos } & \multirow{2}{*}{$\frac{\text { Total }}{\text { No. }}$} \\
\hline & No. & $\%$ & No. & $\%$ & No. & $\%$ & No. & $\%$ & No. & $\%$ & \\
\hline 2001 & 0 & 0 & 5 & 10,2 & 0 & 0 & 41 & 83,7 & 3 & 6,1 & 49 \\
\hline 2002 & 0 & 0 & 0 & 0 & 0 & 0 & 42 & 97,7 & 1 & 2,3 & 43 \\
\hline 2003 & 2 & 3,4 & 0 & 0 & 0 & 0 & 55 & 93,2 & 2 & 3,4 & 59 \\
\hline 2004 & 2 & 3,9 & 1 & 2,0 & 0 & 0 & 45 & 88,2 & 3 & 5,9 & 51 \\
\hline 2005 & 1 & 2,2 & 0 & 0 & 0 & 0 & 43 & 93,5 & 2 & 4,3 & 46 \\
\hline 2006 & 0 & 0 & 1 & 2,5 & 0 & 0 & 38 & 95 & 1 & 2,5 & 40 \\
\hline 2007 & 0 & 0 & 0 & 0 & 0 & 0 & 41 & 91,1 & 4 & 8,9 & 45 \\
\hline 2008 & 0 & 0 & 0 & 0 & 1 & 3,1 & 28 & 87,5 & 3 & 9,4 & 32 \\
\hline 2009 & 1 & 4,5 & 0 & 0 & 0 & 0 & 17 & 77,3 & 4 & 18,2 & 22 \\
\hline 2010 & 0 & 0 & 1 & 4,7 & $3^{a}$ & 14,3 & 14 & 66,7 & 3 & 14,3 & 21 \\
\hline Total & 6 & 1,5 & 8 & 2,0 & 4 & 1,0 & 364 & 89,2 & 26 & 6,3 & 408 \\
\hline
\end{tabular}

a Incluye los dos aislamientos resistentes a rifampicina, descritos en este estudio.

los 10 aislamientos (figura 1). Los aislamientos 1847 y 1852 eran idénticos entre sí y relacionados a la cepa control 1103 (C:2b:P1.5). El aislamiento 1648 (con resistencia intermedia a rifampicina) también fue idéntico a otro aislamiento sensible a rifampicina de 2010 (1841) del mismo fenotipo B:7.1:NST. Los cinco aislamientos restantes presentaban perfiles únicos y fenotipos diferentes.

La resistencia a rifampicina entre cepas de $N$. meningitidis ha sido reconocida esporádicamente en el mundo, generalmente como consecuencia de la quimioprofilaxis administrada a los contactos (3). En el primer par de casos descritos en este estudio, se disponía únicamente del aislamiento relacionado con el caso secundario, por lo que podría corresponder a una situación similar a la descrita anteriormente. Por el contrario, en el segundo par, el aislamiento correspondiente al caso índice ya era altamente resistente a rifampicina. En consecuencia, podría marcar el inicio de la diseminación de un clon que mostró un comportamiento altamente virulento.

La primera medida de prevención aplicada fue la emisión de un comunicado a los laboratorios de microbiología clínica del país, para alertar sobre la situación y recomendar que se realizaran las pruebas de susceptibilidad a rifampicina y ciprofloxacina antes de enviar el aislamiento al laboratorio nacional, ya que este último no podría obtener resultados en un plazo adecuado para cambiar la pauta de la quimioprofilaxis.

La caracterización de los dos aislamientos determinó que ellos eran fenotípica y genotípicamente idénticos, es decir, ambos del serogrupo B, serotipo/serosubtipo 2a:P1.5 y mostraban el

\section{FIGURA 1. Perfiles de electroforesis en gel con campo pulsátil de cepas de Neisseria meningitidis generados por Nhel}

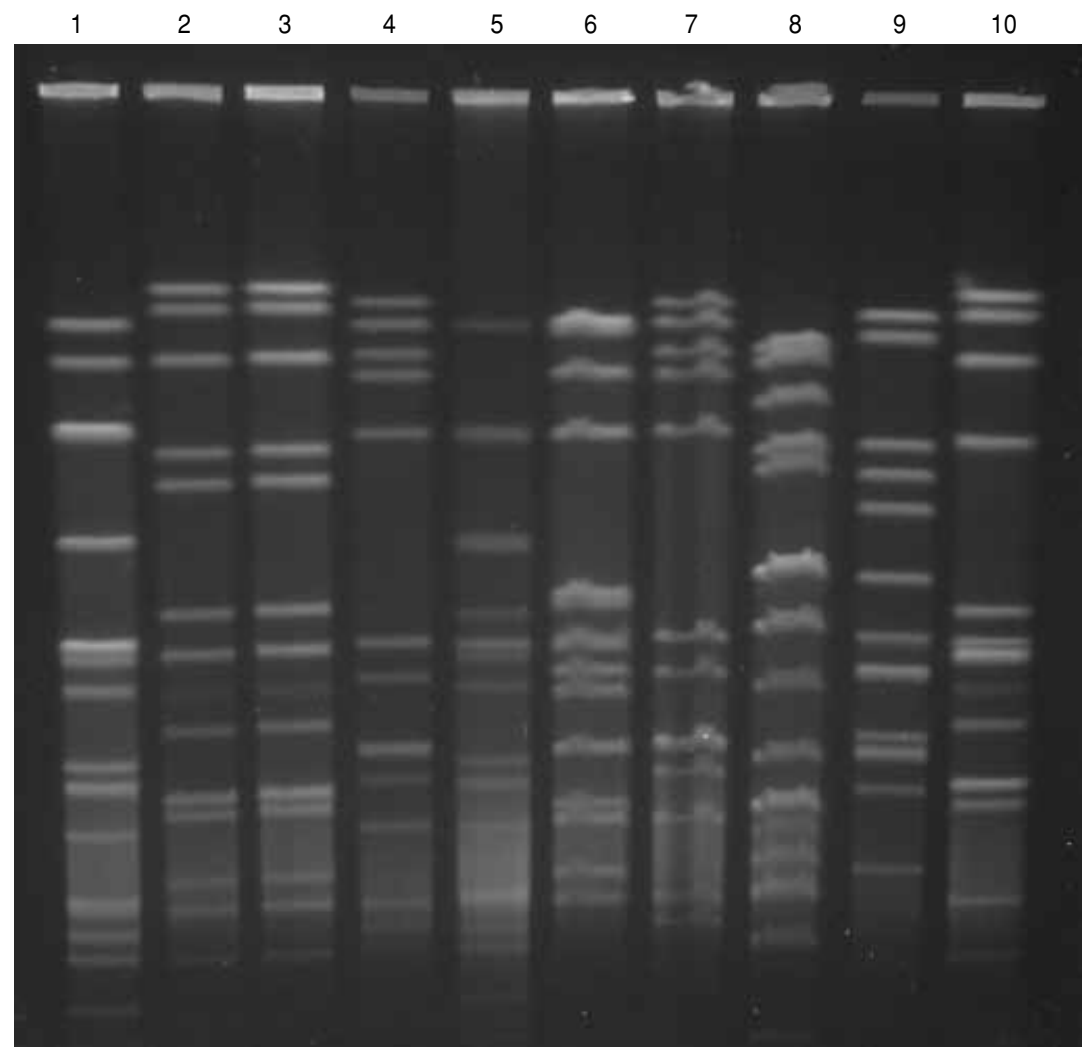

Carril 1: cepa de referencia H44/76, B:15:P1.7,16. Carriles 2 y 3: aislamientos 1847 y 1852, B:2a:P1.5, resistentes a rifampicina, carril 4: aislamiento 1325, B:15:P1.16, resistente a rifampicina. Carril 5: aislamiento 1648, B:7,1:NST, intermedio a rifampicina. Carriles 6: aislamiento 1848, B:7,1.NST. Carril 7: 1841, B:7,1:NST. Carril 8: aislamiento 1845, B:7:NST. Carril 9: 1853, C:NT:NST. Carril 10: cepa control 1103, C:2b:P1.5.

mismo grado de resistencia a rifampicina (CIM $\geq 32 \mu \mathrm{g} / \mathrm{ml})$.

Las cepas de $N$. meningitidis pueden experimentar cambio de serogrupo por transferencia horizontal de genes, fenómeno que se ha observado en situaciones epidémicas y no epidémicas (11). El serotipo/serosubtipo 2a:P1.5, que fuera inicialmente descrito en aislamientos del serogrupo $\mathrm{C}$, se ha identificado progresivamente como causa de brotes epidémicos del serogrupo B, fundamentalmente en aquellos países que han incorporado las vacunas $C$ conjugadas en sus esquemas corrientes de vacunación. En España se han notificado tres brotes por N. meningitidis B:2a:P1.5 desde que la vacuna C conjugada fuera incorporada en 2000. Los 
casos por B:2a:P1.5 notificados presentaron una tasa de letalidad y una frecuencia de casos secundarios mayor que la de los casos ocasionados por otros meningoco$\cos \mathrm{B}$, aunque estas diferencias no son estadísticamente significativas (12).

En el Uruguay, se realizó en 1996 una campaña de vacunación con vacuna A-C polisacárida en respuesta a un brusco incremento en el número de casos de meningitis bacterianas ocasionado por la diseminación de dos clones de N. meningitidis del serogrupo $\mathrm{C}$ en la década de 1990 (10). Entre 2001 y 2002, se realizó otra campaña de vacunación con vacuna B-C, también en respuesta a un brote en la ciudad de Santa Lucía, cercana a Montevideo. Esa vacuna B-C se registró para ser comercializada en 1992, y se administró a un número desconocido de niños y adolescentes.

En el período analizado (2001-2010), los aislamientos del serogrupo $C$ fueron escasos en relación con los del grupo $\mathrm{B}$, y en su mayoría correspondieron al serotipo/serosubtipo 2a:P1.5 (6 de 14 aislamientos), lo cual sugiere una mayor eficacia de la vacuna para prevenir la enfermedad meningocócica causada por el serogrupo C. Es posible entonces que estas vacunas hayan podido ejercer presión inmunológica suficientemente fuerte para seleccionar las variantes B del serotipo/serosubtipo 2a:P1.5.

La caracterización genotípica por PFGE demostró la identidad total de los dos aislamientos B:2a:P1.5, con un pulsotipo único no compartido con ninguno de los otros aislamientos estudiados.
Aparentemente, su perfil de bandas era idéntico al pulsotipo de los aislamientos de un brote ocurrido en España en 2002, identificado como ST11/complejo 37 por tipificación de frecuencias de multisitio exacto o MLST, que comprendía cepas de los serogrupos B y C (10). Estos resultados sugieren otra hipótesis: la introducción de las cepas B:2a:P1.5 al país desde países de otras regiones, como España, ya que la diseminación intercontinental había sido descrita anteriormente.

La cepa 1103, utilizada como control por ser prototipo del clon Paysandú de 1995, era C:2b:P1.5 y similar a la que circuló en Argentina en el mismo período (13). Esta cepa se consideró genéticamente relacionada por PFGE, dado que presentaba una diferencia de tres bandas con el pulsotipo de las cepas B:2a:P1.5. Los aislamientos del mismo fenotipo analizados por MLST fueron asignados al complejo ET-37 o al conglomerado A4 (14). En Italia, en 2000, se notificó un brote causado por una cepa probablemente originada en la Argentina, C:2b:P1.5 con un ST1860 no descrito hasta ese momento, que era una variante en un único alelo del ST11/complejo ET-37 (15). Estos resultados sugieren que tanto los aislamientos resistentes a rifampicina de 2010 como la cepa prototipo del clon Paysandú corresponderían al complejo ET-37. La determinación del secuenciotipo de los aislamientos analizados en este trabajo, así como la secuenciación del gen $r p o B$, que confiere resistencia a rifampicina, permitirán profundizar el conocimiento sobre el origen de estas cepas.

\section{REFERENCIAS}

1. Purcell B, Samuelsson S, Hahné SJM, Ehrhard I, Heuberger S, Camaroni I, et al. Effectiveness of antibiotics in preventing meningococcal disease after a case: systematic review. BMJ. 2004;328:1339-44.

2. Carter PE, Abadi FJR, Yakubu DE, Pennington TH. Molecular characterization of rifampinresistant Neisseria meningitidis. Antimicrob Agents and Chemoter. 1994;38:1256-61.

3. Taha MK, Zarantonelli ML, Ruckly C, Giorgini D, Alonso JM. Rifampin-resistant Neisseria meningitidis. Emerg Infect Dis. 2006; 12:859-60.

4. Rainbow J, Cebelinski E, Bartkus J, Glennen A, Boxrud D, Lynfield R. Rifampin-resistant meningococcal disease. Emerg Infect Dis. 2005;11:977-9.

5. Gabastou JM, Agudelo CI, de Cunto Brandileone MC, Castañeda E, Silva de
Lemos AP, Di Fabio JL, et al. Caracterización de aislamientos invasivos de $S$. pneumoniae, $H$. influenzae y $N$. meningitidis en América Latina y el Caribe: SIREVA II, 2000-2005. Rev Panam Salud Publica. 2008; 24(1):1-15

6. Clinical and Laboratory Standards Institute. Performance Standards for Antimicrobial Susceptibility Testing; Twentieth Informational Supplement 2010. M100-S20.

7. Abdillahi H, Poolman JT. Whole-cell ELISA for typing Neisseria meningitidis with monoclonal antibodies. FEMS Microbiol Lett. 1987; 48:367-71.

8. Popovic T, Schmink S, Rosenstein NA, Ajello GW, Plikaytis B, Hunter SB, et al. Evaluation of pulsed-field gel electrophoresis in epidemiological investigations of meningococcal disease outbreaks caused by Neisseria
En conclusión, los dos eventos de transmisión descritos en este trabajo fueron causados por cepas de N. meningitidis resistentes a rifampicina, con un fenotipo que ya había sido asociado con la gravedad y letalidad de la enfermedad $\mathrm{y}$ un genotipo relacionado con brotes en los que se ha demostrado cambio de cápsula del serogrupo C al B. La falta de conexión epidemiológica directa aparente entre esos dos eventos sugiere una cierta diseminación y la existencia de portadores asintomáticos. La aparición de los casos coincidió con la disminución estacional natural de la incidencia de la enfermedad meningocócica, que antecede al verano uruguayo. Este trabajo describe una situación potencialmente problemática, que requiere fortalecer y extremar la vigilancia de laboratorio de los aislamientos de $N$. meningitidis en el país y la Región de las Américas.

No obstante, los meningococos resistentes a rifampicina se han notificado solo en forma esporádica en el mundo $\mathrm{y}$, hasta el momento, no han sido asociados con brotes ni hiperendemias. Por lo tanto, debe evaluarse cautelosamente el cambio de fármaco antimicrobiano para la profilaxis de los contactos. En el Uruguay el uso de rifampicina está restringido y se utiliza casi exclusivamente para la profilaxis de meningitis y el tratamiento de pacientes con tuberculosis. En la situación epidemiológica actual no se justifica un cambio de medicamento antimicrobiano, a menos que se identifique un caso secundario. meningitidis serogroup C. J Clin Microbiol. 2001;39:75-85.

9. Piet JR, Huis in 't Veld RAG, van Schaik BDC, van Kampen AHC, Baas F, van de Beek D, et al. Genome sequence of Neisseria meningitidis serogroup B strain H44/76. J. Bacteriol. 2011. doi: 10.1128/JB.01331-10.

10. Perez-Trallero E, Vicente D, Montes M, Cisterna R. Positive effect of meningococcal $C$ vaccination on serogroup replacement in Neisseria meningitidis. Lancet. 2002;360:953.

11. Vogel U, Claus H, Frosch M. Rapid serogroup switching in Neisseria meningitidis. N Engl J Med. 2000;342:219-20.

12. Castilla J, Vázquez JA, Salcedo C, García Cenoz M, García Irure JJ, Torroba L, et al. B:2a:P1.5 Meningococcal strains likely arisen from capsular switching event still spreading in Spain. J Cli Microbiol. 2009;47:463-5. 
13. García Gabarrot G, Perez Giffoni G, Camou T. Epidemiología molecular de Neisseria meningitidis serogrupo C (1993-2006). Archiv Pediatr. Uruguay. 2008;79:113-9.

14. Alcalá B, Arreaza L, Salcedo C, Uría MJ, de la Fuente L, Vázquez JA. Capsule switching among C:2b:P1.2,5 meningococcal epidemics alter mass immunization campaign, Spain. Emerg Infect Dis. 2002;8:1512-4.

15. Stefanelli P, Fazio C, Neri A, Tonino S, Mastrantonio P. First report of capsule replacement among electrophoretic type 37
Neisseria meningitidis strains in Italy. J Clin Microbiol. 2003;41:5783-6.

Manuscrito recibido el 10 de abril de 2011. Aceptado para publicación, tras revisión, el 8 de septiembre de 2011.

ABSTRACT The objective of this study was to characterize the phenotype and genotype of two isolates of rifampicin-resistant Neisseria meningitidis associated with two independent events involving transmission of severe meningococcal meningitis that occurred

Detection of rifampicinresistant strains of Neisseria meningitidis in Uruguay in September and October 2010 in Montevideo, Uruguay. The most recent 10 years of data from the national antimicrobial resistance surveillance system were reviewed to estimate the frequency of the particular meningococcal features that were characterized. Rifampicin resistance was studied using the epsilometer test. The serotype and serosubtype of the isolates were determined by ELISA, and the genotype was characterized using DNA digestion with Nhel and pulse field gel electrophoresis. The two isolates were identical: B:2a:P1.5. In the collection of 408 strains of $N$. meningitidis isolated in Uruguay in the past 10 years, the phenotype only appeared in two isolates, which were sensitive to rifampicin. The two isolates studied also shared a single pulse type, which was different from that of two other rifampicinresistant isolates obtained in 2003 and 2007. Consequently, it was concluded that both cases of transmission were caused by a single rifampicin-resistant strain, which could have been an import from another country or else the result of a drift from serogroup $C$ to $B$ due to selective pressure exerted by vaccines administered to the population. It is essential to maintain and maximize surveillance. However, since this type of finding has been sporadic so far, unless a secondary case is identified, there is no justification for changing the antimicrobial drug currently being administered to contacts as prophylaxis.

Key words Neisseria meningitidis; rifampin; meningitis; drug resistance; drug resistance, microbial; Uruguay. 